\title{
Statistical analysis of the
}

SFB

lifetime of diamond

823

impregnated tools for core

drilling of concrete

$\square$
$\mathcal{O}$
$\mathcal{C}$
$\mathcal{O}$
$\mathcal{O}$

Nadja Malevich, Christine H. Müller, Michael Kansteiner, Dirk Biermann, Manuel Ferreira, Wolfgang Tillmann

Nr. 30/2018

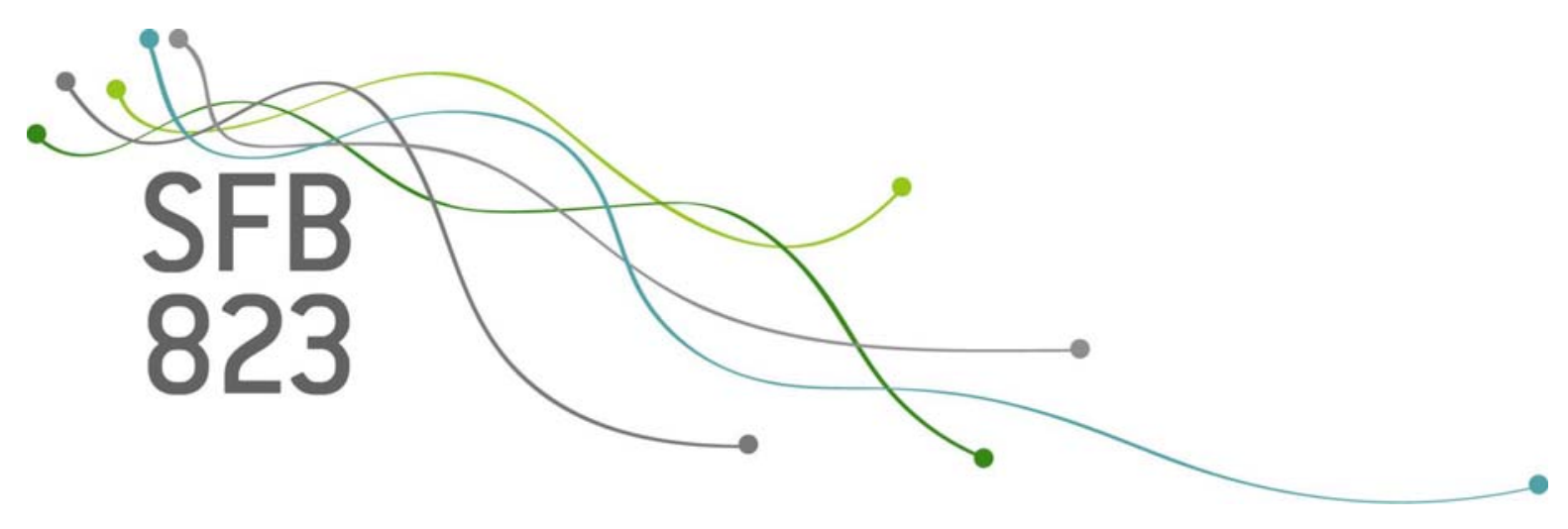





\title{
Statistical analysis of the lifetime of diamond impregnated tools for core drilling of concrete
}

\author{
Nadja Malevich, Christine H. Müller, Michael Kansteiner, Dirk Biermann, Manuel \\ Ferreira and Wolfgang Tillmann
}

\begin{abstract}
The lifetime of diamond impregnated tools for core drilling of concrete is studied via the lifetimes of the single diamonds on the tool. Thereby, the number of visible and active diamonds on the tool surface is determined by microscopical inspections of the tool at given points in time. This leads to interval-censored lifetime data if only the diamonds visible at the beginning are considered. If also the lifetimes of diamonds appearing during the drilling process are included then the lifetimes are doubly interval-censored. A statistical method is presented to analyse the interval-censored data as well as the doubly interval-censored data. The method is applied to three series of experiments which differ in the size of the diamonds and the type of concrete. It turns out that the lifetimes of small diamonds used for drilling into conventional concrete is much shorter than the lifetimes when using large diamonds or high strength concrete.
\end{abstract}

Nadja Malevich (responsible for statistical methods)

Department of Statistics, TU University Dortmund, e-mail: nadja.malevich@tu-dortmund.de

Christine Müller (responsible for statistical methods)

Department of Statistics, TU University Dortmund, e-mail: cmueller@statistik.tu-dortmund.de

Michael Kansteiner (responsible for experimental setup)

Institute of Machining Technology, TU University Dortmund, e-mail: kansteiner@isf.de

Dirk Biermann (responsible for experimental setup)

Institute of Machining Technology, TU University Dortmund, e-mail: biermann@isf.de

Manuel Ferreira (responsible for experimental setup)

Institute of Materials Engineering, TU University Dortmund, e-mail: manuel.ferreira@udo.edu

Wolfgang Tillmann (responsible for experimental setup)

Institute of Materials Engineering, TU University Dortmund, e-mail: wolfgang.tillmann@udo.edu 


\section{Introduction}

Diamond impregnated tools for concrete drilling are so-called "self-sharpening tools". This means that these tools need to wear down within the application so that at any time of the process new sharp diamonds are exposed at the tool surface. Several authors already analysed the wear behaviour of diamond impregnated tools, e.g. [12], [3], [20], [6], and the main wear mechanisms seem to be identified. Nevertheless, extensive scientific work has to be undertaken to increase the process knowledge and understanding to allow further improvements in tool performance [9]. Statistical approaches for the analysis of the wear behaviour of diamond impregnated tools were used in [10], [14] and [4]. But these articles focused on tools for sawing applications of rock. Only a few authors are dealing with the diamond core drilling process [2], [8].

In particular, the wear of the single diamonds in core drilling tools is not studied well up to now although the wear of the tool depends heavily on it. Having an estimate for the lifetime of the single diamonds, we can estimate, for example, the time until a certain number of diamonds are broken out. This, in return, gives us insight into the total lifetime of the drilling tool. Hence, this paper deals especially with the statistical analysis of the lifetime of these single diamonds, where the lifetime of a diamond is understood as the time until the diamond is completely broken out.

One of the challenges of the statistical analysis is that the lifetime of the diamonds cannot be observed directly. The drilling process must be interrupted to check how many diamonds on the tool are broken out. This can be done only at predetermined inspection times. Hence, the lifetimes are given as so-called interval-censored data where only intervals are known in which the exact lifetimes are falling.

The statistical analysis of interval-censored data, also called grouped data, is quite an old research area, see for example the book [11] from 1961. Nevertheless it is of high actual interest which is shown by the new books [1] of 2018 and [16] of 2006 and many recent publications such as [5], [7], [17], [18], [19]. See also [13] for determining optimal inspection times for interval-censored data.

Another challenge is that the drilling tool consists of a metal matrix where the diamonds are embedded. Not all diamonds embedded in this matrix are visible and active in the beginning. Several of them appear only during the drilling process. Hence, the starting point of the lifetimes of these diamonds is not the beginning of the drilling process. Because of the predetermined inspection times, the exact starting points of these lifetimes are not known. They are also interval-censored. Hence, both start and end of the lifetimes are interval-censored so that those data are called doubly interval-censored.

There are not many statistical methods for doubly interval-censored data. The most of them concern nonparametric methods for discrete lifetime distributions with finite support or complicated semiparametric methods for proportional and additive hazard models for the case of covariates, see [16]. Since we are mainly interested in the prediction of lifetimes of diamonds and diamond impregnated tools, we present and apply a rather simple parametric approach based on the exponential distribution. 
The description of the experimental setup is given in Section 2. Thereby, the state of the art of diamond impregnated tools is given before the experiments are described. The statistical methods are presented in Section 3. At first, we provide the method for interval-censored lifetime data with identical known starting times. After this, our method for doubly interval-censored lifetime data is derived. For both situations, confidence sets for the unknown parameter are provided. Based on these confidence sets, confidence sets for related quantities are derived. These quantities include - among other quantities - the expected lifetime and the time until half of the initially visible diamonds are broken out. Section 4 provides the results for the experiments. They show in particular a significant difference between tools with small and large diamonds applied to the conventional concrete. A significant difference appears also between the tools with small diamonds applied to the conventional concrete and a high strength concrete. However, both significances appear only if the diamonds visible in the beginning are used, i.e. for the interval-censored data. The tendency is similar if also the doubly interval-censored data are used, but the differences are not significant anymore. A discussion of these results is given in Section 5 .

\section{Experimental setup}

\subsection{Diamond impregnated tools}

Diamond impregnated metal matrix composites are mainly used as cutting and grinding tools for high abrasive mineral materials in the natural stone and construction industry. Especially, due to the high hardness of granite, basalt and high performance concrete, high demands on the efficiency of the cutting tools exist. To guarantee a high cutting performance monocrystalline synthetic diamonds with an average grain size of $250 \mu \mathrm{m}-500 \mu \mathrm{m}$ were used. These abrasive components are embedded into a metal matrix, which basically consisted of pure cobalt, cobalt/copper or cobalt/bronze until the 2000s. Due to the estimated carcinogenic properties and the rising world market price in particular, most diamond tool manufacturers tend to substitute cobalt with iron, copper and bronze. In terms of important materials characteristics like hardness and wear resistance, the new developed cobalt reduced and cobalt free alternative binder systems reached comparable properties as the wellknown cobalt based matrix compositions. Additionally, higher amount of copper or bronze enables the possibility to adjust the hardness of the metal matrix to the requirements of the machined mineral subsoil. Depending on the hardness and abrasiveness of the present mineral materials (concrete, basalt), the metal matrix composition has to be adapted to guarantee a suitable grain protrusion to avoid the negative influence of rounding effects on the diamond grains. 


\subsection{Production of diamond impregnated tools}

The small diamond grinding segments, which were attached to drill bits, saw blades or wire saws by brazing or laser welding, are fabricated in a powder metallurgical process route. The first step is the premixing of the chosen metal powder components with 5-10 vol-\% synthetic monocrystalline diamond grains. Subsequently, the homogenized powder blend is cold pressed and the obtained green bodies are sintered to achieve an almost pore-free structure with an optimal final strength. The sintering procedure is conducted pressureless in a vacuum or inert gas furnace or in a hot-pressing facility which heats and mechanically presses the green bodies simultaneously in a graphite mold. In contrast to vacuum sintering, the hot-pressing process leads to lower porosity with a significant reduction of the sintering time. Besides these conventional sintering methods, the new developed field assisted sintering technology (FAST) is similar to the hot-pressing but offers the possibility to increase the heating rate up to $\max .1000 \mathrm{~K} / \mathrm{min}$. This is realized by a direct current up to $60 \mathrm{kA}$ and a pulse time of a few milliseconds, which leads to small plasma arcs between neighboring powder particles resulting in a very fast partial heating of the powder greenbody.

\subsection{The experiments}

The experiments were conducted on a 3-axis machine centre and a special tool holder for a single segment with a diameter of $d=100 \mathrm{~mm}$ was used, see Fig.1 a). The workpieces made of two different types of concrete were clamped on a force dynamometer for force measurements.

Concrete is made of aggregates which means stones, cement and water. Hence, concrete has an inhomogeneous material structure. The classification of concrete is based on its compressive strength. Within the tests, two types of concrete were used: a high strength concrete with a compressive strength of C100/115 and a conventional concrete with compressive strength of C20/25. C100/115 is used for example as material for foundations of skyscrapers whereas $\mathrm{C} 20 / 25$ is used for buildings without special requirements. The main aggregate material of concrete C100/115 is basalt. In C20/25, the main aggregates are stones from the Rhine river, see Fig. $1 \mathrm{~b}$ ). Hence, in comparison, the $\mathrm{C} 20 / 25$ concrete possess harder and more brittle material phases than $\mathrm{C} 100 / 115$. But the cement phase in C20/25 has got a smaller hardness and reveals more open pores. In contrast, the cement phase of $\mathrm{C} 100 / 115$ is quite dense.

The concrete workpieces had a volume of $b=150 \mathrm{~mm} \times 1=150 \mathrm{~mm} \times \mathrm{h}$ $=150 \mathrm{~mm}$ and were mounted on top of the dynamometer, see Fig.1. In order to focus on the wear behaviour of the segments, the tested parameters for the segments were kept constant. The drilling depth was $\mathrm{s}=4 \mathrm{~mm}$, the infeed velocity was $\mathrm{v}_{\mathrm{f}}=4 \mathrm{~mm} / \mathrm{min}$ and the circumferential speed was $\mathrm{v}_{\mathrm{u}}=3,225 \mathrm{~m} / \mathrm{s}$. Overall, each segment achieved a cumulated drilling depth of $\mathrm{s}_{\mathrm{tot}}=200 \mathrm{~mm}$. The infeed velocity 
and the circumferential speed were deduced from force-controlled core drilling tests with eight segments and the same segment specifications. In order to remove slurry from the process, water with an additive was used (Bechem Avantin 361, concentration $\mathrm{p}=7 \%$ ).

The tested segments were of laboratory dimensions (height $\mathrm{h}_{\mathrm{seg}}=10 \mathrm{~mm}$, width $\mathrm{w}_{\mathrm{seg}}=5 \mathrm{~mm}$ and length $1_{\text {seg }}=10 \mathrm{~mm}$ ). The metal matrix was Diabase V21 which main components are iron, copper, cobalt and tin. The diamonds were of quality Syngrit SDB 1055 by Element Six. The grit size of the diamonds was $d_{k}=40 / 50$ mesh and $d_{k}=20 / 30$ mesh. The diamond concentration $c=C 20$ was used.

In the following chapters, the segments with the diamond grit size $d_{k}=20 / 30$ mesh are named B18 and B19, and the segments with the diamond grit size $d_{k}=40 / 50$ mesh are referred to as B28 and B29. B18 and B29 were used on concrete C100/115, and $\mathrm{B} 19$ and $\mathrm{B} 28$ on $\mathrm{C} 20 / 25$.

This provided four sequences of experiments where the experiments of a sequence were done under the same conditions. Each experiment of these sequences is given by the drilling of depth $\mathrm{s}=4 \mathrm{~mm}$ which means a drilling time of $1 \mathrm{~min}$. Since the cumulated drilling depth of each sequence is $\mathrm{s}_{\mathrm{tot}}=200 \mathrm{~mm}$, each sequence consists of 50 experiments and a cumulated drilling time of $50 \mathrm{~min}$.

After each experiment, the tested segment was microscopically inspected using a digital microscope (DigiMicro Profi by DNT). Hence, the inspection times are given by the drilling depth of $\mathrm{s}=4 \mathrm{~mm}$ and correspond to one minute of drilling. The microscopical analysis comprised the counting of the number of exposed dia-

a)

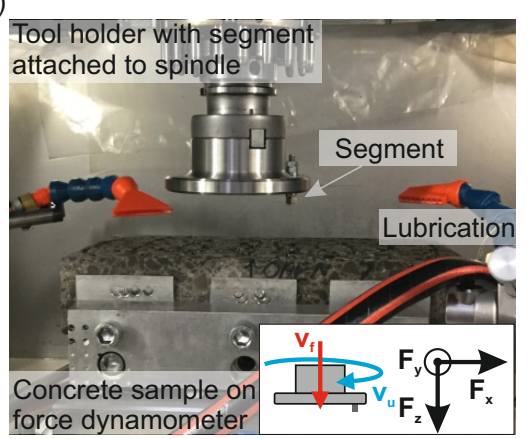

c)

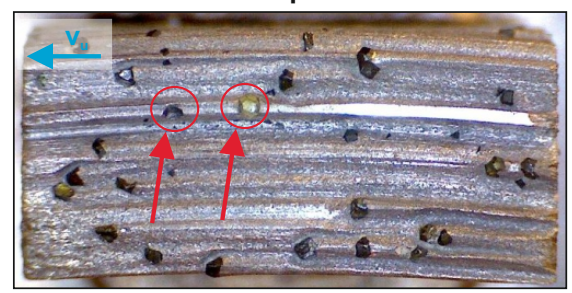

b)

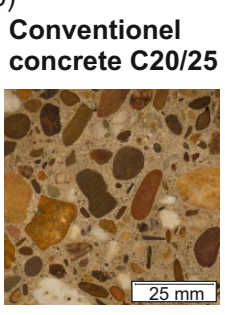

Cement CEM I 32,5 R Aggregates sand $0 / 2$, stone from river Rhine 2/8 and 8/16

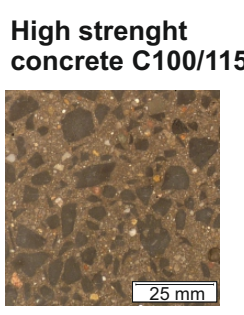

Cement CEM I 52,5 R Aggregates sand $0 / 2$, basalt $2 / 8$ and $8 / 16$

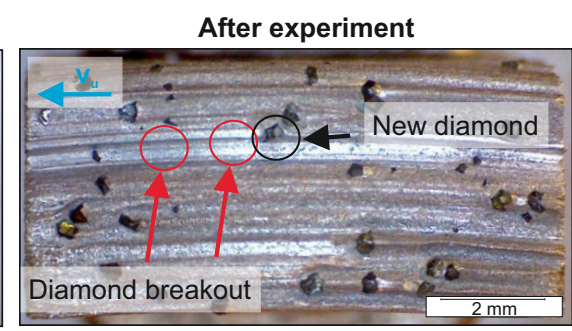

Fig. 1 a) Experimental setup for single segment tests b) Concrete workpieces c) Microscopic pictures of diamond impregnated segments with diamond breakout 
monds on the segment surface. Thereby, each exposed diamond got a label so that the visibility of the single diamonds could be followed over the experiments. For simplification, it was only distinguished between visible " 1 " and non-visible diamonds " 0 ". There was no differentiation between flat/worn diamonds or diamonds with partial outbreak respectively. Only when a complete diamond breakout occurred, this diamond was classified as " 0 ". Diamonds which are not visible in the beginning are also classified as "0" until they become visible. Fig.1 c) shows the surface of a segment before and after the experiment where two diamonds broke out (marked by red circles) and one new diamond occurred (marked by a black circle).

\section{Statistical methods for interval-censored lifetime data}

\subsection{Analysis of lifetimes with the same starting times}

Let $T_{1}, \ldots, T_{N}$ be independent and identically distributed nonnegative random variables (lifetime variables of $N$ objects) with the cumulative distribution function $F_{\theta}$, where $\theta \in \mathbb{R}^{d}$ is an unknown parameter. However, the realizations $t_{1}, \ldots, t_{N}$ of $T_{1}, \ldots, T_{N}$ are not observed. The objects are only observed at given fixed times $0=\tau_{0}<\tau_{1}<\ldots<\tau_{I}<\tau_{I+1}=\infty$, which are called inspection times. This means that only realizations $z_{n}$ of $Z_{n}$ with

$$
Z_{n}=i, \quad \text { if } \quad T_{n} \in\left(\tau_{i-1}, \tau_{i}\right], \quad i=1, \ldots, I+1,
$$

are observed for $n=1, \ldots, N$. Such data are called interval-censored data. The goal is to estimate the unknown parameter $\theta$ and to construct the corresponding confidence intervals.

The likelihood function for interval-censored lifetimes $z_{1}, \ldots, z_{N}$ can be found as follows:

$$
\begin{aligned}
l(\theta) & :=l\left(\theta ; z_{1}, \ldots, z_{N}\right)=\prod_{n=1}^{N} P_{\theta}\left(Z_{n}=z_{n}\right) \\
& =\prod_{n=1}^{N} \prod_{i=1}^{I+1} P_{\theta}\left(T_{n} \in\left(\tau_{i-1}, \tau_{i}\right]\right)^{\mathbb{1}}\left\{z_{n}=i\right\}=\prod_{n=1}^{N} \prod_{i=1}^{I+1}\left(F_{\theta}\left(\tau_{i}\right)-F_{\theta}\left(\tau_{i-1}\right)\right)^{\mathbb{1}_{\left\{z_{n}=i\right\}} .}
\end{aligned}
$$

Maximising the likelihood function $l(\theta)$, we obtain the maximum likelihood estimate $\widehat{\theta}$ of $\theta$ :

$$
\widehat{\theta}=\arg \max \left\{l(\theta) ; \theta \in \mathbb{R}^{d}\right\} .
$$

Then, an asymptotic $(1-\alpha)$-confidence interval for $\theta$ based on the likelihood-ratio test is given by 


$$
\mathscr{C}\left(z_{1}, \ldots, z_{N}\right)=\left\{\theta \in \mathbb{R}^{d} ;-2 \ln \left(\frac{l\left(\theta ; z_{1}, \ldots, z_{N}\right)}{l\left(\widehat{\theta} ; z_{1}, \ldots, z_{N}\right)}\right) \leq \chi_{d ; 1-\alpha}^{2}\right\},
$$

where $l$ is the likelihood function, $\widehat{\theta}$ is the maximum likelihood estimate for $\theta$ and $\chi_{d ; 1-\alpha}^{2}$ is the $(1-\alpha)$-quantile of the chi-square distribution with $d$ degrees of freedom.

For our specific situation with the lifetimes of diamonds we consider an exponential model, i.e. $T_{1}, \ldots, T_{N} \sim \operatorname{Exp}(\lambda)$, where $\lambda>0$ is unknown. The likelihood function in this case is

$$
l(\lambda)=\prod_{n=1}^{N} \prod_{i=1}^{I}\left(e^{-\lambda \tau_{i-1}}-e^{-\lambda \tau_{i}}\right)^{\mathbb{1}_{\left\{z_{n}=i\right\}}}\left(e^{-\lambda \tau_{I}}\right)^{\left.\mathbb{1}_{\{z n}=I+1\right\}}
$$

and the asymptotic $(1-\alpha)$-confidence interval for $\lambda$ is

$$
\mathscr{C}\left(z_{1}, \ldots, z_{N}\right)=\left\{\lambda \in \mathbb{R} ;-2 \ln \left(\frac{l\left(\lambda ; z_{1}, \ldots, z_{N}\right)}{l\left(\widehat{\lambda} ; z_{1}, \ldots, z_{N}\right)}\right) \leq \chi_{1 ; 1-\alpha}^{2}\right\} .
$$

\subsection{Analysis of lifetimes with different interval-censored starting times}

Let $D_{1}, \ldots, D_{N}$ be independent and identically distributed nonnegative random variables (death times of $\mathrm{N}$ objects), but here with different starting times (times of birth) $B_{1}, \ldots, B_{N}$ so that lifetime variables $T_{1}:=D_{1}-B_{1}, \ldots, T_{N}:=D_{N}-B_{N}$ are independent identically distributed nonnegative random variables with the cumulative distribution function $F_{\theta}$, where $\theta \in \mathbb{R}^{p}$ is an unknown parameter. We assume also that $B_{1}, \ldots, B_{N}, T_{1}, \ldots, T_{N}$ are independent. However, neither the realizations $d_{1}, \ldots, d_{N}$ of $D_{1}, \ldots, D_{N}$ nor the realizations $b_{1}, \ldots, b_{N}$ of $B_{1}, \ldots, B_{N}$ are observed. With given inspection times $0=\tau_{0}<\tau_{1}<\ldots<\tau_{I}<\tau_{I+1}=\infty$, we observe only whether $d_{n}$ and $b_{n}$ are lying in $\left(\tau_{i-1}, \tau_{i}\right], i=1, \ldots, I+1$, or not. This means that only realizations $\boldsymbol{z}_{n}$ of $\boldsymbol{Z}_{n}$ with

$$
Z_{n}=(h, i), \quad \text { if } \quad B_{n} \in\left(\tau_{h-1}, \tau_{h}\right], \quad D_{n} \in\left(\tau_{i-1}, \tau_{i}\right], \quad h<i, \quad h, i=1, \ldots, I+1,
$$

are observed for $n=1, \ldots, N$. Such data are called doubly interval-censored data. The goal is, as before, to estimate the unknown parameter $\theta$ and to construct the corresponding confidence intervals.

The likelihood function for interval-censored birth and death data $z_{1}, \ldots, z_{N}$ is given by 


$$
\begin{aligned}
l(\theta) & :=l\left(\theta ; z_{1}, \ldots, z_{N}\right)=\prod_{n=1}^{N} P_{\theta}\left(\boldsymbol{Z}_{n}=\boldsymbol{z}_{n}\right)=\prod_{n=1}^{N} \prod_{\substack{h, i=1 \\
h<i}}^{I+1} P_{\theta}\left(\boldsymbol{Z}_{n}=(h, i)\right) \\
& =\prod_{n=1}^{N} \prod_{i=1}^{I+1} \prod_{\substack{h=1 \\
h<i}}^{I+1} P_{\theta}\left(B_{n} \in\left(\tau_{h-1}, \tau_{h}\right], D_{n} \in\left(\tau_{i-1}, \tau_{i}\right]\right)^{\mathbb{1}_{\left\{z_{n}=(h, i)\right\}}} .
\end{aligned}
$$

Since $T_{n}:=D_{n}-B_{n}, n=1, \ldots, N$, we can rewrite

$$
\begin{aligned}
P_{\theta} & \left(B_{n} \in\left(\tau_{h-1}, \tau_{h}\right], D_{n} \in\left(\tau_{i-1}, \tau_{i}\right]\right)=P_{\theta}\left(B_{n} \in\left(\tau_{h-1}, \tau_{h}\right], B_{n}+T_{n} \in\left(\tau_{i-1}, \tau_{i}\right]\right) \\
& =\iint_{\mathbb{R}^{2}} \mathbb{1}_{\left(\tau_{h-1}, \tau_{h}\right]}\left(y_{1}\right) \mathbb{1}_{\left(\tau_{i-1}, \tau_{i}\right]}\left(y_{2}\right) d P_{\theta}^{\left(B_{n}, B_{n}+T_{n}\right)}\left(y_{1}, y_{2}\right) .
\end{aligned}
$$

Using the elementary transformation theorem from the measure theory and then the independence of $B_{n}$ and $T_{n}$, we obtain

$$
\begin{aligned}
\iint_{\mathbb{R}^{2}} & \mathbb{1}_{\left(\tau_{h-1}, \tau_{h}\right]}\left(y_{1}\right) \mathbb{1}_{\left(\tau_{i-1}, \tau_{i}\right]}\left(y_{2}\right) d P_{\theta}^{\left(B_{n}, B_{n}+T_{n}\right)}\left(y_{1}, y_{2}\right) \\
& =\iint_{\mathbb{R}^{2}} \mathbb{1}_{\left(\tau_{h-1}, \tau_{h}\right]}(u) \mathbb{1}_{\left(\tau_{i-1}, \tau_{i}\right]}(v+u) d P_{\theta}^{\left(B_{n}, T_{n}\right)}(u, v) \\
& =\iint_{\mathbb{R}^{2}} \mathbb{1}_{\left(\tau_{h-1}, \tau_{h}\right]}(u) \mathbb{1}_{\left(\tau_{i-1}, \tau_{i}\right]}(v+u) d P_{\theta}^{T_{n}}(v) d P_{\theta}^{B_{n}}(u) \\
& =\int_{\tau_{h-1}}^{\tau_{h}} \int_{\tau_{i-1}-u}^{\tau_{i}-u} d F_{\theta}(v) d G_{\theta}(u)=\int_{\tau_{h-1}}^{\tau_{h}}\left(F_{\theta}\left(\tau_{i}-u\right)-F_{\theta}\left(\tau_{i-1}-u\right)\right) d G_{\theta}(u),
\end{aligned}
$$

where $F_{\theta}$ is the distribution function of $T_{1}, \ldots, T_{N}$, and $G_{\theta}$ is the distribution function of $B_{1}, \ldots, B_{N}$. This implies

$$
l(\theta)=\prod_{n=1}^{N} \prod_{i=1}^{I+1} \prod_{\substack{h=1 \\ h<i}}^{I+1}\left(\int_{\tau_{h-1}}^{\tau_{h}}\left(F_{\theta}\left(\tau_{i}-u\right)-F_{\theta}\left(\tau_{i-1}-u\right)\right) d G_{\theta}(u)\right)^{\mathbb{1}_{\left\{\boldsymbol{z}_{n}=(h, i)\right\}}}
$$

Without any further assumptions on the distribution function $G_{\theta}$, the likelihood function can not be found explicitly.

For our specific situation with newborn diamonds, we consider the following model: $B_{1}, \ldots, B_{N} \sim \operatorname{Exp}\left(\lambda_{0}\right)$ with unknown $\lambda_{0}>0$ and $T_{1}, \ldots, T_{N} \sim \operatorname{Exp}(\lambda)$ with unknown $\lambda>0$, so that $\theta=\left(\lambda_{0}, \lambda\right)$. Using the method described in Section $3.1-$ now for interval-censored birth times - we obtain the estimate $\widehat{\lambda}_{0}$ for $\lambda_{0}$ and assume that $B_{1}, \ldots, B_{N} \sim \operatorname{Exp}\left(\widehat{\lambda}_{0}\right)$. Then, a plug-in likelihood function of (2) can be calculated as follows:

$l(\lambda)=\prod_{n=1}^{N} \prod_{i=1}^{I+1} \prod_{\substack{h=1 \\ h<i}}^{I+1}\left(\frac{\widehat{\lambda}_{0}}{\lambda-\widehat{\lambda}_{0}}\left(e^{-\lambda \tau_{i-1}}-e^{-\lambda \tau_{i}}\right)\left(e^{\left(\lambda-\widehat{\lambda}_{0}\right) \tau_{h}}-e^{\left(\lambda-\widehat{\lambda}_{0}\right) \tau_{h-1}}\right)\right)^{\mathbb{1}_{\left\{z_{n}=(h, i)\right\}}}$

Using the plug-in likelihood function $l(\lambda)$, we obtain the plug-in maximum likelihood estimate $\widehat{\lambda}$ and a plug-in confidence interval for $\lambda$ using (1). This plug-in 
confidence interval is an asymptotic confidence interval since $\widehat{\lambda}_{0}$ is a consistent estimator for $\lambda_{0}$.

\subsection{Analysis of lifetimes with mixed starting times}

In some situations, we have the combination of the models presented in 3.1 and 3.2. For example, in drilling experiments we had some diamonds visible at the beginning and some diamonds, which appeared later during the drilling. For this case we use the following model.

Let the birth times of the diamonds visible at the beginning be modeled by $B_{1}=\cdots=B_{N_{0}}=0$ and the birth times of the new diamonds be modeled by the independent and identically distributed random variables $B_{N_{0}+1}, \ldots, B_{N}, N_{0}<N$, with the common distribution function $G_{\theta}$, where $\theta \in \mathbb{R}^{p}$ is unknown. Let $T_{1}, \ldots, T_{N}$ be the lifetime random variables (also independent and identically distributed) with the common distribution function $F_{\theta}$. We assume that $B_{1}, \ldots, B_{N}, T_{1}, \ldots, T_{N}$ are independent. We do not observed the realizations $B_{N_{0}+1}, \ldots, B_{N}$ and the realizations of $T_{1}, \ldots, T_{N}$. Only realizations $\boldsymbol{z}_{n}$ of $\boldsymbol{Z}_{n}$ with

$Z_{n}=\left\{\begin{array}{l}(0, i), \text { if } B_{n}=0, T_{n} \in\left(\tau_{i-1}, \tau_{i}\right], \quad i=1, \ldots, I+1, \\ (h, i), \text { if } B_{n} \in\left(\tau_{h-1}, \tau_{h}\right], B_{n}+T_{n} \in\left(\tau_{i-1}, \tau_{i}\right], \quad h<i, \quad h, i=1, \ldots, I+1,\end{array}\right.$

are observed for $n=1, \ldots, N$.

Similar as in Sections 3.1 and 3.2, we get the likelihood function for the data $z_{1}, \ldots, z_{N}$ in the following form:

$$
\begin{aligned}
& l(\theta)= \prod_{n=1}^{N} \prod_{i=1}^{I+1} P_{\theta}\left(T_{n} \in\left(\tau_{i-1}, \tau_{i}\right]\right)^{\mathbb{1}_{\left\{z_{n}=(0, i)\right\}} .} \\
& \cdot \prod_{\substack{h=1 \\
h<i}}^{I+1} P_{\theta}\left(B_{n} \in\left(\tau_{h-1}, \tau_{h}\right], B_{n}+T_{n} \in\left(\tau_{i-1}, \tau_{i}\right]\right)^{\mathbb{1}}\left\{z_{n}=(h, i)\right\} \\
&=\prod_{n=1}^{N} \prod_{i=1}^{I+1}\left(F_{\theta}\left(\tau_{i}\right)-F_{\theta}\left(\tau_{i-1}\right)\right)^{\mathbb{1}_{\left\{z_{n}=(0, i)\right\}} .} \\
& \cdot \prod_{\substack{h=1 \\
h<i}}^{I+1}\left(\int_{\tau_{h-1}}^{\tau_{h}}\left(F_{\theta}\left(\tau_{i}-u\right)-F_{\theta}\left(\tau_{i-1}-u\right)\right) d G_{\theta}(u)\right)^{\mathbb{1}_{\left\{z_{n}=(h, i)\right\}}} .
\end{aligned}
$$

If we assume that $\theta=\left(\widehat{\lambda}_{0}, \lambda\right)$ with $\widehat{\lambda}_{0}$ defined in Section 3.2, $G_{\theta}=\operatorname{Exp}\left(\widehat{\lambda}_{0}\right)$ and $F_{\theta}=\operatorname{Exp}(\lambda)$, we obtain 


$$
\begin{aligned}
l(\lambda)= & \prod_{n=1}^{N} \prod_{i=1}^{I+1}\left(e^{-\lambda \tau_{i-1}}-e^{-\lambda \tau_{i}}\right)^{\mathbb{1}_{\left\{\boldsymbol{z}_{n}=(0, i)\right\}}} \\
& \cdot \prod_{\substack{h=1 \\
h<i}}^{I}\left(\frac{\widehat{\lambda}_{0}}{\lambda-\widehat{\lambda}_{0}}\left(e^{-\lambda \tau_{i-1}}-e^{-\lambda \tau_{i}}\right)\left(e^{\left(\lambda-\widehat{\lambda}_{0}\right) \tau_{h}}-e^{\left(\lambda-\widehat{\lambda}_{0}\right) \tau_{h-1}}\right)\right)^{\mathbb{1}_{\left\{\boldsymbol{z}_{n}=(h, i)\right\}}}
\end{aligned}
$$

Using the likelihood function $l(\lambda)$, we obtain the maximum likelihood estimate $\widehat{\lambda}$ and an asymptotic confidence interval for $\lambda$ from (1).

\subsection{Confidence sets for related quantities}

Usually, the parameter $\lambda$ of the exponential distribution itself is not of interest. The following quantities are of more interest when analyzing the lifetime of diamond impregnated tools:

(a) expected lifetime of each diamond;

(b) expected number of breakouts in $N$ diamonds within $[0, T]$;

(c) time $T_{L, N}$ so that the probability of a breakout of at least $L$ of $N$ diamonds equals $p$.

Let us consider each of these quantities.

(a) Expected lifetime of each diamond is

$$
q_{1}(\lambda):=E_{\lambda}\left(T_{n}\right)=\frac{1}{\lambda}
$$

since we assume $T_{1}, \ldots, T_{N} \sim \operatorname{Exp}(\lambda)$.

(b) Probability that one diamond breaks out within $[0, T]$ is

$$
p_{T}:=P_{\lambda}\left(T_{n} \leq T\right)=F_{\lambda}(T)=1-e^{-\lambda T} .
$$

Then, the number $N_{b}$ of breakouts in $N$ diamonds within $[0, T]$ follows the binomial distribution $\operatorname{Bin}\left(N, p_{T}\right)$. Therefore, expected number of breakouts in $N$ diamonds within $[0, T]$ is given by

$$
q_{2}(\lambda):=N p_{T}=N F_{\lambda}(T)=N\left(1-e^{-\lambda T}\right) .
$$

(c) Since $N_{b} \sim \operatorname{Bin}\left(N, p_{T}\right)$ (see (b)), the probability that at least $L$ of $N, L \leq N$, diamonds break out within $[0, T]$ is given by

$$
P_{\lambda}\left(N_{b} \geq L\right)=\sum_{l=L}^{N}\left(\begin{array}{c}
N \\
l
\end{array}\right)\left(1-e^{-\lambda T}\right)^{l} e^{-\lambda T(N-l)} .
$$


Time $T_{L, N}$ so that the probability of a breakout of at least $L$ of $N$ diamonds is 0.5 can be found by solving the following equation:

$$
\sum_{l=L}^{N}\left(\begin{array}{c}
N \\
l
\end{array}\right)\left(1-e^{-\lambda T_{L, N}}\right)^{l} e^{-\lambda T_{L, N}(N-l)}=0.5
$$

Note that the function on the left of the equation is monotone increasing with respect to $\lambda T_{L, N}$ for all $L, N$ with $L \leq N$. This means, there is a unique solution of the equation and it has the following form:

$$
q_{3}(\lambda):=T_{L, N}(\lambda)=\frac{f(L, N)}{\lambda}
$$

where $f(L, N)$ is a function of $L, N$ and can be found numerically for each $L, N$.

All quantities $q_{i}(\lambda), i=1,2,3$, can be estimated by replacing $\lambda$ with its estimate $\hat{\lambda}$, i.e. by $q_{i}(\hat{\lambda})$. Since $q_{1}(\lambda)$ and $q_{3}(\lambda)$ are decreasing functions with respect to $\lambda$, the confidence interval for $q_{i}(\lambda), i=1,3$, is given by

$$
\left[q_{i}\left(\widehat{\lambda}_{u}\right), q_{i}\left(\widehat{\lambda}_{l}\right)\right]
$$

where $\mathscr{C}\left(z_{1}, \ldots, z_{N}\right)=\left[\widehat{\lambda}_{l}, \widehat{\lambda}_{u}\right]$ is the confidence interval for $\lambda$. Similarly, since $q_{2}$ is increasing in $\lambda$, the confidence interval for $q_{2}(\lambda)$ is $\left[q_{2}\left(\widehat{\lambda}_{l}\right), q_{2}\left(\widehat{\lambda}_{u}\right)\right]$.

\section{Results}

The statistical analysis was done by $\mathrm{R}$ [15].

Within the conducted experiments, one or more diamond breakouts were observed for segments B28, B29, and B19. In contrast, segment B18 showed no complete diamond breakout. Hence, a lifetime analysis for single diamonds is not possible here and it was decided to neglect this segment for the analysis. Further experiments need to be conducted for this segment to get enough data for lifetime estimation.

\subsection{Using only diamonds visible in the beginning}

The maximum likelihood estimates for $\lambda$ and the corresponding asymptotic $98.4 \%$ confidence intervals for three experimental setups (B19, B28, B29) are given in Table 1 and Figure 2. Note that the level $\alpha$ is chosen so that $(1-\alpha)^{3} \geq 0.95$. It is because of the adjustment by testing (with level $95 \%$ ) the hypothesis that the lifetime parameter $\lambda$ is the same for B19, B28 and B29. Figure 2 shows that the confidence intervals have an empty intersection. This allows us to reject the null hypothesis and 
conclude that there is significant difference between all three experimental setups. At the same time, the chosen level $98.4 \%$ for confidence intervals allows to compare the experiments in pairs (with level al least 95\%). The null hypotheses about the pairwise equality of the lifetime parameters are rejected when the corresponding confidence intervals are disjoint. So, we see that there is no significant difference between B19 and B29, since the intersection is not empty. However, B28 distinguishes significantly from B19 and B29.

\begin{tabular}{|c|c|c|}
\hline Experiment & ML-estimate of $\lambda$ & $98.4 \%$-confidence interval \\
\hline B19 & 0.00489 & {$[0.00083,0.01519]$} \\
B28 & 0.03310 & {$[0.01734,0.05638]$} \\
B29 & 0.00448 & {$[0.00152,0.00988]$} \\
\hline
\end{tabular}

Table 1 ML-estimates and $98.4 \%$-confidence intervals for $\lambda$

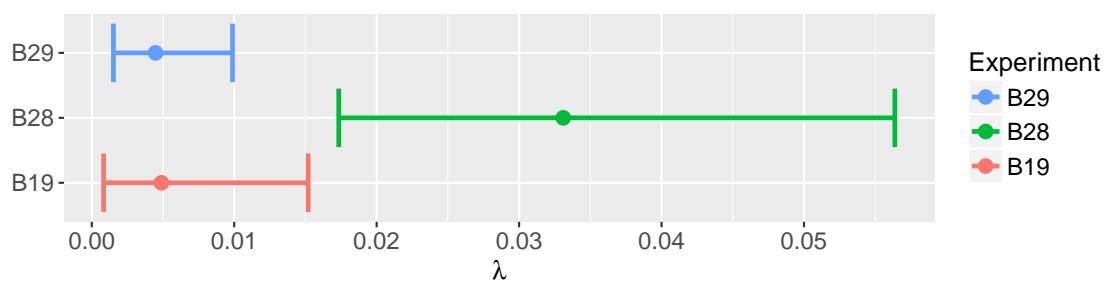

Fig. 2 ML-estimates and 98.4\%-confidence intervals for $\lambda$

The estimates and the confidence intervals for the quantities represented in Section 3.4 are given below. Figure 3 provides the estimates for the expected lifetime $1 / \lambda$ of each diamond. Figure 4 gives the estimated expected number of breakouts of initially visible diamonds within the time interval $[0,50]$ (in min). Figure 5 represents $\widehat{T}_{L, N}$ for $L=N / 2$ and $L=N$, i.e. the estimated time at which with probability 0.5 at least half (all, respectively) of the initially visible diamonds are broken out. Note that the number $N$ of diamonds visible at the beginning are different for B19, B28 and B29 (14, 22 and 33, respectively) so that the confidence intervals for B28 and B29 in Figure 4 and for B19 and B28 in Figure 5 are not disjunct.

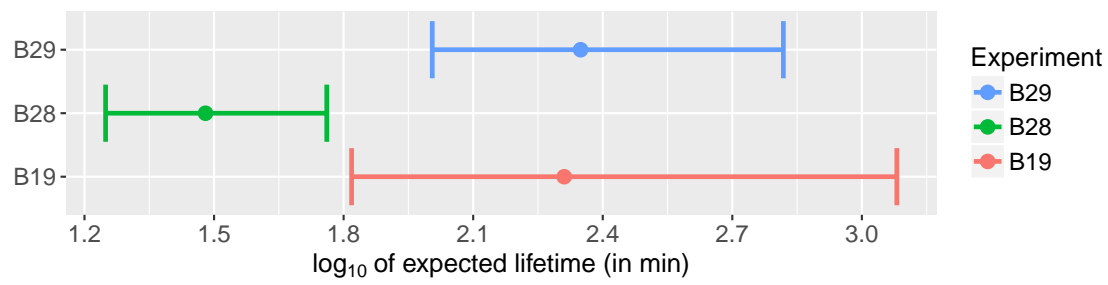

Fig. 3 ML-estimates and 98.4\%-confidence intervals for expected lifetime $1 / \lambda$ 


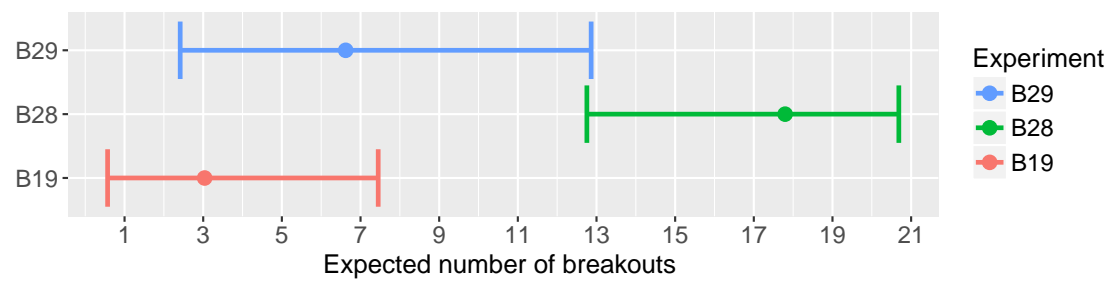

Fig. 4 ML-estimates and 98.4\%-confidence intervals for the expected number of breakouts of initially visible diamonds within the time interval $[0,50]$ (in min)

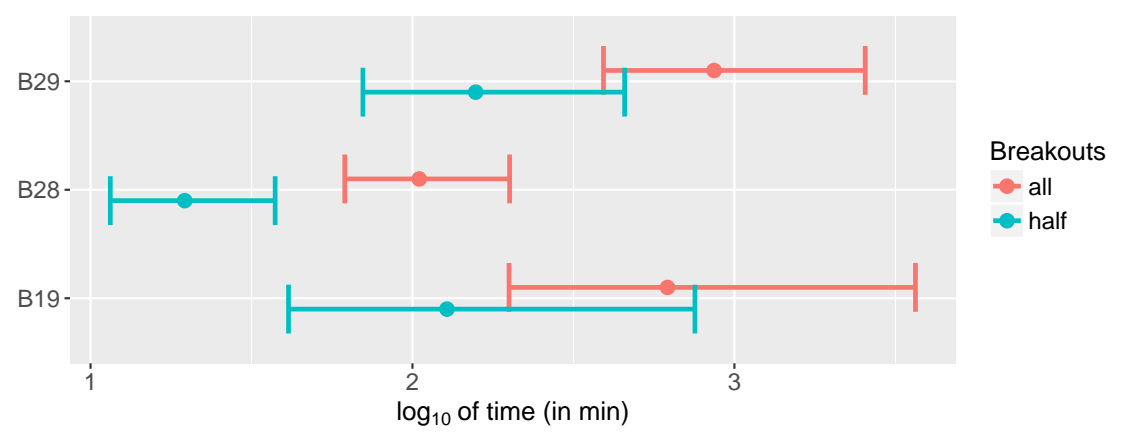

Fig. 5 ML-estimates and 98.4\%-confidence intervals for the times $T_{L, N}$ with $L=N / 2$ and $L=N$

\subsection{Using all active diamonds}

The maximum likelihood estimation for the birth time parameter $\lambda_{0}$ from the model represented in Section 3.2 yields the following values:

$$
\text { B19: } \widehat{\lambda}_{0}=0.054, \quad \text { B28: } \widehat{\lambda}_{0}=0.052, \quad \text { B29: } \widehat{\lambda}_{0}=0.049 \text {. }
$$

These estimates and the corresponding asymptotic $98.4 \%$-confidence intervals for $\lambda_{0}$ are given in Figure 6. Note that the lengths of the intervals are different because of the different number of new diamonds that appeared during the drilling in B19, B28 and B29 (9, 46 and 22, respectively).

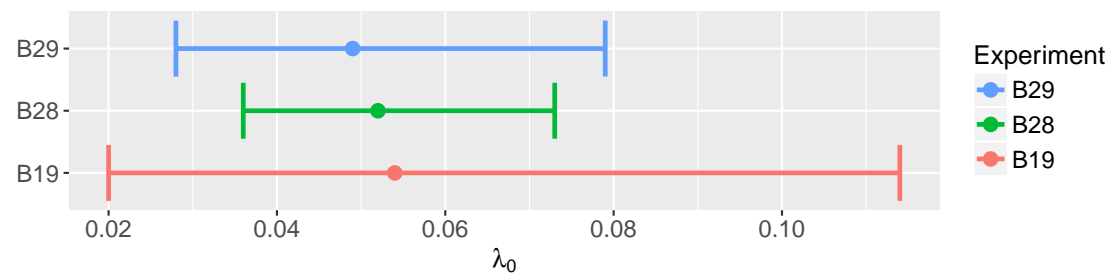

Fig. 6 ML-estimates and 98.4\%-confidence intervals for $\lambda_{0}$ 
The maximum likelihood estimates for the lifetime parameter $\lambda$ and the corresponding asymptotic $98.4 \%$-confidence intervals for B19, B28, B29 are given in Table 2 and Figure 7. Note again that the level $\alpha$ is chosen so that $(1-\alpha)^{3} \geq 0.95$ (for explanation see Section 4.1).

Figure 7 shows that the confidence intervals are not disjunct anymore in comparison with the confidence intervals from Figure 2. This means that there is no significant difference between the lifetime parameter $\lambda$ in all three experimental setups. Figure 8 provides the estimates for the expected lifetime $1 / \lambda$ of each diamond.

\begin{tabular}{|c|c|c|}
\hline Experiment & ML-estimate of $\lambda$ & $98.4 \%$-confidence interval \\
\hline B19 & 0.00300 & {$[0.00047,0.00919]$} \\
B28 & 0.00879 & {$[0.00526,0.01346]$} \\
B29 & 0.00302 & {$[0.00114,0.00634]$} \\
\hline
\end{tabular}

Table 2 ML-estimates and $98.4 \%$-confidence intervals for $\lambda$

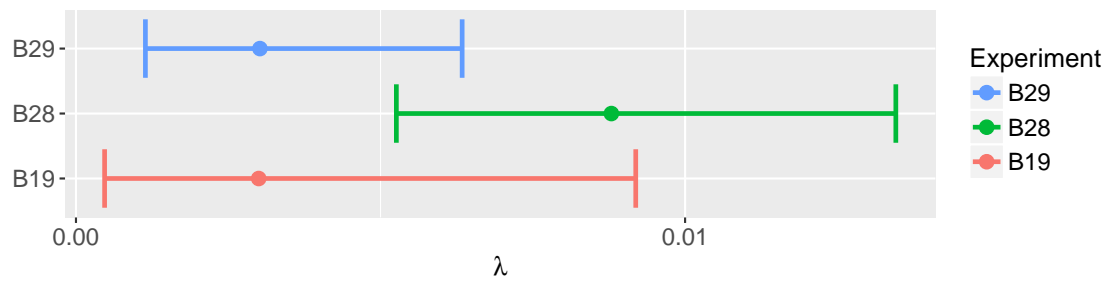

Fig. 7 ML-estimates and 98.4\%-confidence intervals for $\lambda$

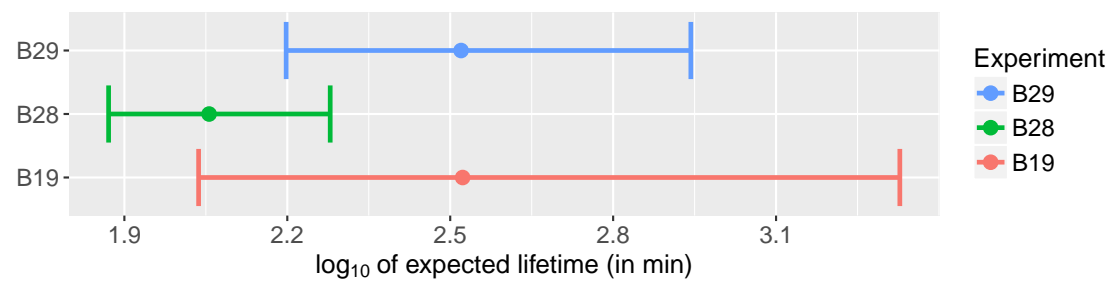

Fig. 8 ML-estimates and $98.4 \%$-confidence intervals for expected lifetime $1 / \lambda$

\section{Discussion}

The wear of diamond impregnated drilling tools was studied via the lifetime of the single active diamonds in three different setups. The three setups concerned small diamonds (grit size 40/50 mesh) applied to conventional concrete (C20/25), small diamonds applied to high strength concrete (C100/115), and large diamonds (grit size 20/30 mesh) applied to conventional concrete. In order to observe the lifetime 
of the single diamonds, the drilling process was stopped every minute and the active diamonds were marked via inspection of the surface of the drilling segment. This led to interval-censored lifetimes for the diamonds visible in the beginning and to doubly interval-censored lifetimes for diamonds appearing during the drilling process.

To analyse these interval-censored data, exponential distributions for the lifetimes and the birth times of diamonds appearing during the drilling process were assumed. Moreover, a simple plug-in method for confidence intervals for the lifetimes in the three experimental setups was used. It turned out that the lifetimes of small diamonds are shorter than those of large diamonds in the tools applied to the conventional concrete. Moreover, the lifetimes of small diamonds in the tools applied to conventional concrete were shorter than those in the tools applied to high strength concrete. These differences were significant if only the diamonds visible in the beginning were used. However, the significance disappeared when using all active diamonds. A reason might be that, in all setups, the estimated expected lifetimes of all active diamonds were longer than the estimated expected lifetimes of diamonds visible in the beginning so that consequently the variance in a model with exponential distributions should be larger. It is not surprising that the estimated expected lifetimes of the initially visible diamonds were shorter since these diamonds were observed over longer period of time and had more chances to break out within this period.

The significance also disappeared when some related quantities are considered. This holds for the expected number of breakouts of the initially visible diamonds within the time interval up to 50 minutes and for the time so that the probability of the breakout of all initially visible diamonds is 0.5 . However, this is due to the different numbers of diamonds visible in the beginning which were 22 and 33 for the small diamonds and 14 for the large diamonds.

More experiments should be used to see whether a significant difference can be observed also for these quantities and for the case of using all active diamonds. Moreover, in future work, the simple plug-in method for confidence intervals should be compared with a method where the two parameters of the two exponential distributions (for lifetimes and birth times) are estimated simultaneously. Additionally, other distributions with more parameters like Weibull or gamma distributions should be studied for the lifetimes and birth times.

\section{Acknowledgments}

The authors gratefully acknowledge support from the Collaborative Research Center "Statistical Modelling of Nonlinear Dynamic Processes" (SFB 823, B4) of the German Research Foundation (DFG). 


\section{References}

1. Bogaerts, K., Komarek, A., Lesaffre, E.: Survival analysis with interval-censored data: a practical approach with examples in R, SAS, and BUGS. Interdisciplinary Statistics Series, Chapman \& Hall/CRC, Boca Raton, (2018)

2. Carpinteri, A., Dimastrogiovanni, L., Pugno, N.: Fractal coupled theory of drilling and wear. International Journal of Fracture 131:131-142, (2005)

3. Di Ilio, A., Togna, A.: A theoretical wear model for diamond tools in stone cutting. International Journal of Machine Tools and Manufacture 43:1171-1177, (2003)

4. Ersoy, A., Buyuksagic, S., Atici, U.: Wear characteristics of circular diamond saws in the cutting of different hard abrasive rocks. Wear 258:1422-1436, (2005)

5. Gao, F., Zeng, D., Couper, D., Lin, D.Y.: (2018) Semiparametric regression analysis of multiple right- and interval-censored events. J Am Stat Assoc., (2018) DOI: 10.1080/01621459.2018.1482756

6. Hu, Y.N., Wang, C.Y., Ding, H.N., Wang, Z.W.: Wear mechanism of diamond saw blades for dry cutting concrete. Key Engineering Materials 304-305:315-319, (2006)

7. Ismail, A.A. (2015) Optimum partially accelerated life test plans with progressively Type I interval-censored data. Sequential Anal 34:135-147, (2015)

8. Kansteiner, M., Biermann, D., Dagge, M., Mller, C., Ferreira, M., Tillmann, W.: Statistical evaluation of the wear behaviour of diamond impregnated tools used for the core drilling of concrete, Proceedings of the EURO PM 2017, Milan, EPMA, (2017)

9. Konstanty, J., Kim, T.-W., Kim, S.-B.: Resistance to abrasive wear of materials used as metallic matrices in diamond impregnated tools. In: Antoniac, I. (Eds.): Materials Science Forum: 1125-1128, (2007)

10. Konstanty, J.S., Tyrala, D.: Wear mechanism of iron-base diamond-impregnated tool composites. Wear 303:533-540, (2013)

11. Kulldorff, G.: Contributions to the theory of estimation from grouped and partially grouped samples. John Wiley \& Sons, New York, (1961)

12. Liao, Y.S., Luo, S.Y.: Wear characteristics of sintered diamond composite during circular sawing. Wear 157:325-337, (1992)

13. Malevich, N., Müller, C.H.: Optimal designs for inspection times of interval-censored data. Submitted, (2018)

14. Özçelik, Y.: Multivariate statistical analysis of the wear on diamond beads in the cutting of andesitic rocks. In: Xipeng, X. (Eds.): Key Engineering Materials. Machining of Natural Stone Materials, Vol. 250:118-130, (2003)

15. R Development Core Team: R: A language and environment for statistical computing. $\mathrm{R}$ Foundation for Statistical Computing, Vienna, Austria, http://cran.r-project.org/ (2017)

16. Sun, J.: The statistical analysis of interval-censored failure time data. Statistics for Biology and Health, Springer, New York, (2006)

17. Tsai, T.-R., Lin, C.-W.: Acceptance sampling plans under progressive interval censoring with likelihood ratio. Stat Papers 51:259-271, (2010)

18. Wang, S., Wang. C., Wang. P., Sun, J.: Semiparametric analysis of the additive hazards model with informatively interval-censored failure time data. Comput Stat Data Anal 125:19, (2018)

19. Wu, S.-J., Huang, S.-R.: Optimal progressive group-censoring plans for exponential distribution in presence of cost constraint. Stat Papers 51:431-443, (2010)

20. Yu, Y.Q., Xu, X.P.: Improvement of the performance of diamond segments for rock sawing, Part 1: Effects of segment components. In: Xipeng, X. (Eds.): Key Engineering Materials. Machining of Natural Stone Materials, Vol. 250:46-53, (2003) 

